\title{
The impact of asymptomatic individuals on the strength of public health interventions to prevent the second outbreak of COVID-19
}

\section{Xiaochen Wang}

Beijing University of Posts and Telecommunications

\section{Shengfeng Wang}

Beijing University of Posts and Telecommunications

\section{Yueheng Lan}

Beijing University of Posts and Telecommunications

\section{Xiaofeng Tao}

Beijing University of Posts and Telecommunications

Jinghua Xiao ( $\nabla$ jhxiao@bupt.edu.cn )

Beijing University of Posts and Telecommunications

\section{Research Article}

Keywords: COVID-19, asymptomatic individuals, SIR-typed model, control measures, second outbreak

Posted Date: May 6th, 2020

DOI: https://doi.org/10.21203/rs.3.rs-27142/v1

License: (9) This work is licensed under a Creative Commons Attribution 4.0 International License. Read Full License

Version of Record: A version of this preprint was published at Nonlinear Dynamics on June 14th, 2020. See the published version at https://doi.org/10.1007/s11071-020-05736-x. 


\title{
The impact of asymptomatic individuals on the strength of public health interventions to prevent the second outbreak of COVID-19
}

\author{
Xiaochen Wang · Shengfeng Wang • \\ Yueheng Lan · Xiaofeng Tao · Jinghua \\ Xiao
}

Received: date / Accepted: date

\begin{abstract}
The pandemic of coronavirus disease 2019(COVID-19) has threatened the social and economic structure all around the world. Generally, COVID19 has three possible transmission routes, including pre-symptomatic, symptomatic, and asymptomatic transmission, among which the last one has brought a severe challenge for the containment of the disease. One core scientific question is to understand the influence of asymptomatic individuals and of the strength of control measures on the evolution of the disease, particularly on a second outbreak of the disease. To explore these issues, we proposed a novel compartmental model that takes the infection of asymptomatic individuals into account. We get the relationship between asymptomatic individuals and critical strength of control measures theoretically. Furthermore, we verify the reliability of our model and the accuracy of the theoretical analysis by using the real confirmed cases of COVID-19 contamination. Our results, showing the importance of the asymptomatic population on the control measures, would provide useful theoretical reference to the policymakers and fuel future studies of COVID-19.
\end{abstract}

Keywords COVID-19, asymptomatic individuals, SIR-typed model, control measures, second outbreak

Xiaochen Wang, Xiaofeng Tao

National Engineering Laboratory for Mobile Network Technologies, Beijing University of Posts and Telecommunications, Beijing 100876, China

Shengfeng Wang, Yueheng Lan, Jinghua Xiao (ه)

School of Science, Beijing University of Posts and Telecommunications, Beijing 100876, China

E-mail: jhxiao@bupt.edu.cn

Yueheng Lan, Jinghua Xiao

State Key Lab of Information Photonics and Optical Communications, Beijing University of Posts and Telecommunications, Beijing 100876, China 


\section{Introduction}

By April 7, 2020, there are 1,279,722 confirmed cases of coronavirus disease 2019 (COVID-19) in the world, and almost all countries or regions are affected[1]. To contain the outbreak of COVID-19, policies including quarantining cities, limiting public activities, extending holidays, are implemented. These control measures have reduced the spreading of COVID-19 among the population but also have caused significant disruption to the social and economic structure. Therefore, in the study of epidemiological models, on one hand, it is needed to predict the evolution of COVID-19; on the other hand, it should be helpful to access how long these control measures should last and to what extent these control measures should keep. Different models may alter the predictions of the epidemic and help plan the control measures.

According to the reported data, recent researches estimate the epidemiological parameters and predict the trends of COVID-19. Guan el al.[2] analyzes the clinical characteristics of laboratory-confirmed COVID-19 patients from China to give an estimation of epidemiological parameters, including the basic reproductive number, which is usually used to determine if an infectious disease can outbreak[3], incubation time, etc. Shao et al.[4] proposed a series of time delay dynamical systems, and they estimated the reproductive number of COVID-19 based on Wallinga and Lipsitch framework[5]. Shi et al.[6] used the SEIR model, considering the small transmission rate of the incubation period to fit the data and predict the peak number. Yang et al. [7] used the modified SEIR model, considering the strict measures and analyzing the risk of the second outbreak of the disease.

Such SIR-typed models are based on the understanding of the potential transmission routes of the epidemic. The transmission of COVID-19 has three possible routes [8]. The first one is the symptomatic transmission, which refers to the transmission from a person while they are experiencing symptoms. The second one is the pre-symptomatic transmission. It refers to the transmission from a pre-symptomatic case that can occur before symptom onsets. The third one is the asymptomatic transmission, which refers to the transmission of the virus from a person who does not develop symptoms. The identification of pre-symptomatic and asymptomatic individuals are mainly through contact tracing efforts and enhanced investigation of clusters of confirmed cases[9]. It leaves asymptomatic individuals infectious for an extended period, which would change the macroscopic spreading picture of the epidemic.

To tackle this issue, in this paper, we consider a novel compartmental model called SALIR epidemiological model which analyzes the influence of asymptomatic individuals and of the strength of control measures. In detail, when a healthy individual is infected, we assume that he or she would be transformed into an asymptomatic state with probability $\alpha$, otherwise into a pre-symptomatic state with probability $1-\alpha$. Also, we introduce a parameter $m$ in our model to represent the strength of control measures. From the clinical characteristic of the COVID-19, some parameters in our model are given, and we consider the impact of $\alpha$ on the evolution of the epidemic. Furthermore, 
when the epidemic tends to decline, we analyze the critical strength of control measures which take the asymptomatic individuals into account to prevent a second outbreak of the COVID-19 related epidemic.

\section{SALIR Model Formulation}

We use a novel compartmental model called SALIR model, including the migration of population to analyze the spreading of COVID-19 on the city and country scale. Conceptually, the SALIR model is shown as in Fig. 1

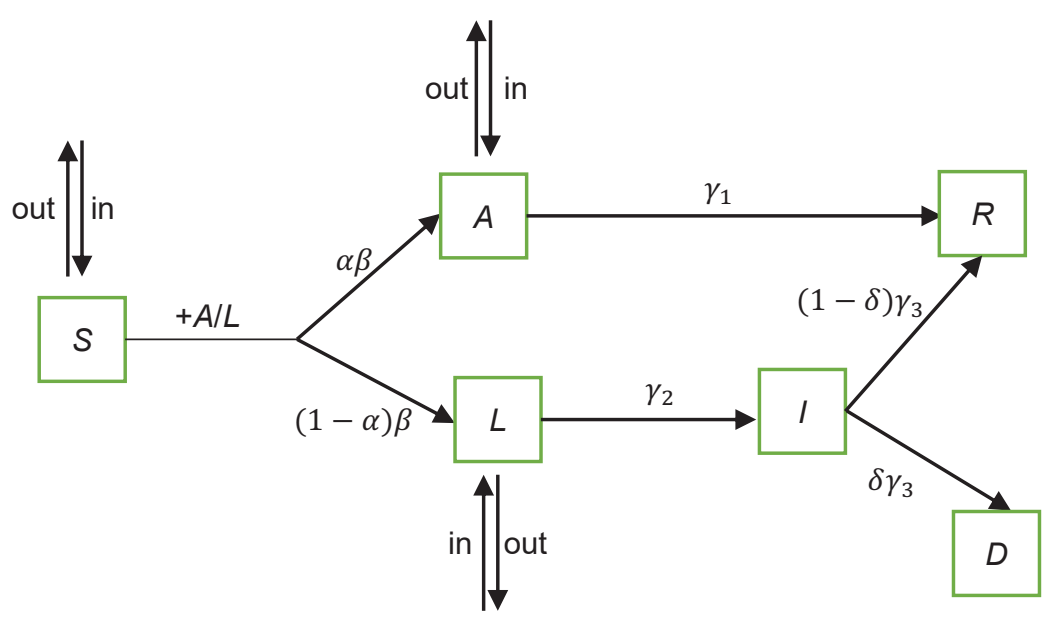

Fig. 1 The sketch map of the SALIR model.

At any time step, an individual is in one of the five states: $S, A, L, I$, and $R$. $D$ represents that an individual dies of COVID-19. We explain these states of Fig. 1 as the following

(1)Individual in the $S$ state is susceptible and can be converted to either $A$ state with probability $\alpha \beta$ or $L$ state with probability $(1-\alpha) \beta$ when contacts with an individual in the $A$ or $L$ state. $\beta$ is the spreading probability of the disease, and $\alpha$ is the probability for the susceptible individual to be in the $A$ state after infection. The number of individuals in the $S$ state at time $t$ is $S(t)$.

(2)Individual in the $A$ state has no symptom of the disease and corresponds to the asymptomatic individual in the epidemiological research. The individual can infect the susceptible individuals with probability $\beta$, and would recover with probability $\gamma_{1}=\frac{1}{t_{1}}$ directly. $t_{1}$ is the duration time of infection for the individuals in the $A$ state. A case study [9] shows that the duration time of infection of an asymptomatic individual is about 19(unite: day) or longer, and thus we assume that the infectious time $t_{1}$ is 21 (unit: day). The number of individuals in the $A$ state at time $t$ is denoted by $A(t)$. 
(3)Individual in the $L$ state is the infected individual before symptoms appear or symptomatic individual before quarantine or hospitalization. The individual in the $L$ state can infect the susceptible individuals with probability $\beta$ and would transform into $I$ state with probability $\gamma_{2}=\frac{1}{t_{2}+t_{3}}$, where $t_{2}$ is the incubation time and equals to 7(unit: day) on average according to the research [2], and $t_{3}$ is the time from symptoms onset to quarantine or hospitalization and equals to 3(unit: day) on average [10]. So, we assume that an individual in the $L$ state would transform into $I$ state with $\gamma_{2}=\frac{1}{7+3}=\frac{1}{10}$. The number of individuals in the $L$ state at time $t$ is denoted by $L(t)$.

(4)Individual in the $I$ state has symptoms but has been quarantined or hospitalized so that he or she cannot infect the other susceptible individuals. The individual in the $I$ state would transform into $R$ state with probability $(1-\delta) \gamma_{3}=(1-\delta) \frac{1}{t_{4}}$, where $\delta$ is the death rate and is close to $0.03[10]$, $t_{4}$ represents the average interval time between the hospitalization and the recovery or death of individuals. According to the clinical characteristic of COVID-19 [2], the time interval between the symptoms onset and recovery or death is 18 days, so we assume that $t_{4}=18-t_{3}=15$ (unit: day) and $\gamma_{3}=\frac{1}{15}$. The number of individuals in the $I$ state is $I(t)$. In addition, the cumulative number of individuals in the $I$ state by time $t$ is denoted by $C_{I}(t)$, which corresponds to the number of confirmed cases in real data.

(5)Individual in the $R$ state represents that the individual has recovered. The number of individuals in the $R$ state at time $t$ is $R(t)$.

Such a compartmental model, to some extent, can be called as a susceptible $(S)$ $\operatorname{asymptomatic}(A)$-latent $(L)$-infected $(I)$-recovered $(R)$ model. However, the latent individual experiences an incubation time and a waiting time of quarantine or hospitalization, and the infected individual has no significant ability to affect healthy individuals because he or she has been quarantined or hospitalized.

Besides the transition rule of the epidemic states, we consider the mobility of individuals as well as the strength of control measures(i.e., public health interventions).

(1)At any time step, individuals in the $S, A$, and $L$ states can move in or out the community, which are represented by $S_{\text {in/out }}, A_{\text {in/out }}$ and $L_{\text {in/out }}$ respectively.

(2)In the situation of disease-free spreading, an individual would leave the $S$ state with probability $\beta$ when contacts with an individual in the $A$ or $L$ states. However, under the implementation of some control measures(e.g., home quarantine, limiting the traffic), the probability would reduce to $m(t) \beta$, where $m(t)$ is the strength of control measures. The stronger the strength is, the smaller the value of $m(t)$ is. 
According to the above descriptions, the discrete equations are as follows

$$
\begin{aligned}
& S(t+1)=S(t)-m(t) \beta \frac{A(t)+L(t)}{N(t)} S(t)+S_{\text {in }}(t)-S_{\text {out }}(t) \\
& A(t+1)=A(t)+\alpha m(t) \beta \frac{A(t)+L(t)}{N(t)} S(t)-\gamma_{1} A(t)+A_{\text {in }}(t)-A_{\text {out }}(t), \\
& L(t+1)=L(t)+(1-\alpha) m(t) \beta \frac{A(t)+L(t)}{N(t)} S(t)-\gamma_{2} L(t)+L_{\text {in }}(t)-L_{\text {out }}(t), \\
& I(t+1)=I(t)+\gamma_{2} L(t)-\gamma_{3} I(t) \\
& R(t+1)=R(t)+\gamma_{1} A(t)+(1-\delta) \gamma_{3} I(t),
\end{aligned}
$$

where $N(t)$ represents the total number of individuals in contact with each other in the community at time $t$, and thus $N(t)=S(t)+A(t)+L(t)+$ $R(t), \frac{A(t)+L(t)}{N(t)}$ is the probability that a susceptible individual contacts with an individual in the $A$ or $L$ state at time $t$.

In our model, parameters $\gamma_{1}, \gamma_{2}, \gamma_{3}$ represent the spontaneous transition of states and thus can be estimated by the clinical data of individuals. Parameter $\beta$ represents the spreading ability of the disease and is usually estimated by its evolution process. Thus, we only need to estimate the value of $\beta$ and $m(t)$ under different $\alpha$ based on the reported cases.

\section{Critical Strength of Control Measures}

This section will explore the critical strength of control measures to contain the epidemic spreading. We assume that the total population size of the community is stable; that is, the outflow of population is approximate to the inflow. The condition that the system in Eq. (1) is stable satisfies $A(t)=0$ or $L(t)=0$, or

$$
\begin{aligned}
\alpha m \beta \frac{A(t)+L(t)}{N(t)} S(t) & =\gamma_{1} A(t), \\
(1-\alpha) m \beta \frac{A(t)+L(t)}{N(t)} S(t) & =\gamma_{2} L(t) .
\end{aligned}
$$

When the value of $A(t)$ and $L(t)$ are extremely small at the initial moment, thus $S(t) \approx N(t)$. Based on the Eq. (2), we can get the critical strength of control measures that prevent the spreading of the epidemic as follows

$$
m_{c}=\frac{\gamma_{1} \gamma_{2}}{\beta\left(\gamma_{1}-\alpha \gamma_{1}+\alpha \gamma_{2}\right)}
$$

It is worth noting that the probability $\alpha$ for the susceptible individual to be in the $A$ state after infection and the parameters of epidemic spreading(i.e., $\beta, \gamma_{1}$ and $\gamma_{2}$ ) all affect the value of $m_{c}$ in Eq. (3). When $\alpha=0, m_{c}=\frac{\gamma_{2}}{\beta}$ conforms to the result of the classical SIR model. 
In the following, we will verify the reliability of the SALIR model and the accuracy of the critical strength analysis by using the real confirmed data of COVID-19.

\section{Numerical Results and Discussions}

From December 2019, a series of pneumonia cases(COVID-19) in the city of Wuhan, in China, has attracted the attention of Chinese health authorities [11]. With the coming of the Spring Festival, a large amount of migrants left Wuhan to return home city or town. To contain the spreading of COVID19, China adopts control measures on January 23, 2020 [7].

To explore the spreading ability of COVID-19 and the strength of control measures needed to contain its spreading, we collect the confirmed data of Wuhan and China[12] and derive the epidemic curve by using the SALIR model. By analyzing the early transmission dynamics in the city of Wuhan[13], we add a gap period of incubation time before the time of illness oneset to simulate the infection of the first patient.

As most of the flow is outflow during Spring Festival in Wuhan according to the statistics [14], the rate of outflow relative to the inflow is represented by $l$. We assume that $l$ is a constant before sealing the city of the January 23, 2020 , and is close to 0 later. In addition, the strength of control measures at time $t$ is denoted by $m(t)$. The stronger the strength is, the smaller the value of $m(t)$ is. $m(t)$ reads as follows

$$
m(t)= \begin{cases}1, & t \leq t_{0}, \\ e^{-\lambda\left(t-t_{0}\right)}, & t_{0}<t<T, \\ m_{0}, & t \geq T,\end{cases}
$$

where $t_{0}$ represents the day of January 22, 2020, which is the day before the control measures were implemented in Wuhan. Under the strict control, the spreading of the disease gradually declined. $T$ is assumed the day that the strength of the control measures is reduced to rescue the economy of the city.

In the initial stage of COVID-19 spreading, there are likely many undetected cases[15,16]. With the adoption of clinical detection on February 12, 2020, the number of confirmed cases increases substantially, which can basically reflect the real situation. Thus, we use the least square method to compare the cumulative number $C_{I}(t)$ of individuals in the $I$ state with the real confirmed data from February 12, 2020, to April 7, 2020, to estimate the parameters of the SALIR model.

Considering the presence of asymptomatic individuals, we estimate the spreading probability $\beta$ and $\lambda$, and explore the robust of the model with different $\alpha$. In Fig. 2(a),(b),(c), and (d), we find that the numerical results of the SALIR model are in good agreement with the real data from February 12, 2020 to April 7, 2020. Moreover, the estimated value of spreading probability is near 0.3 when $\alpha$ is small. Based on the estimated spreading probability, the basic 

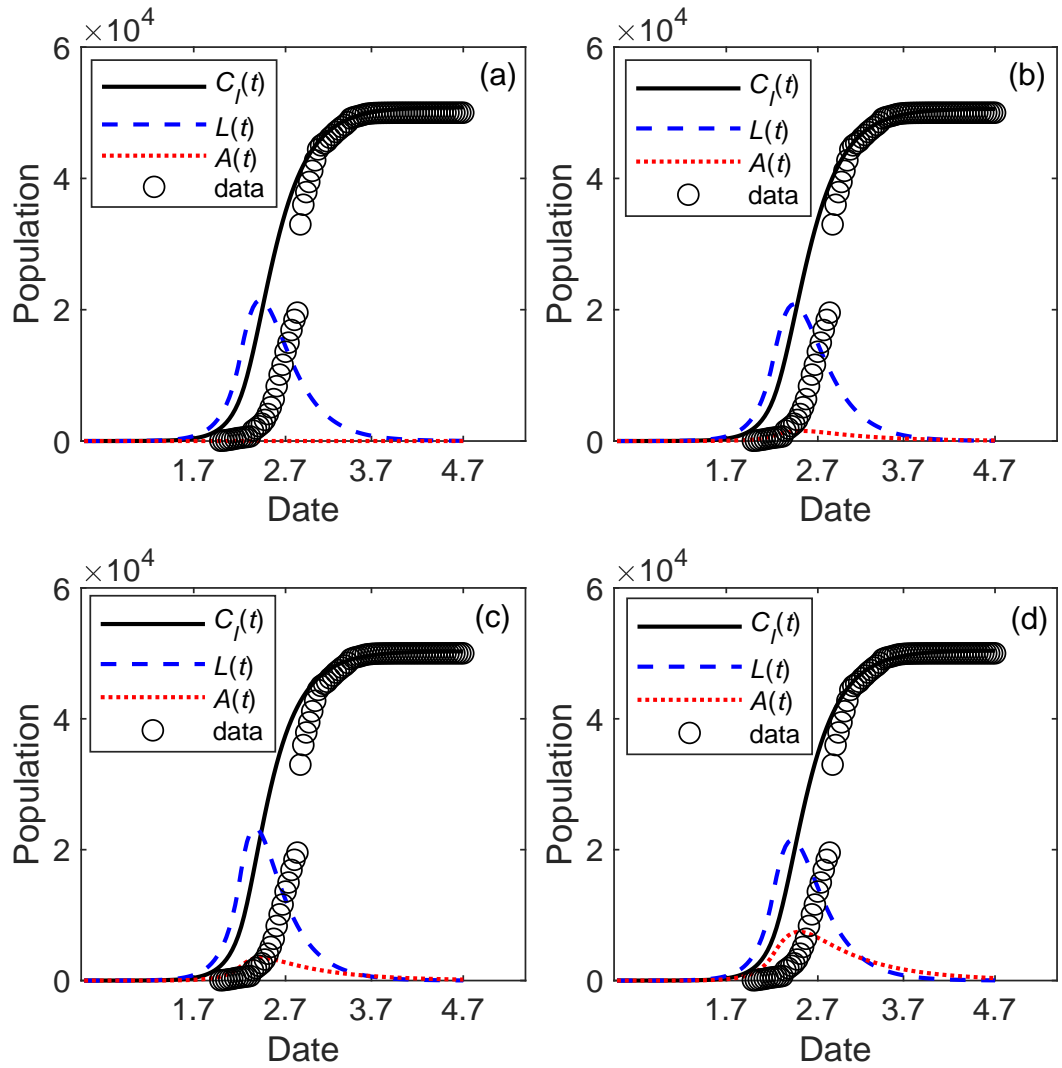

Fig. 2 The fitted epidemic curve with different $\alpha^{\prime} s$. The estimated parameters are (a) $\beta=$ $0.305, \lambda=0.16$ when $\alpha=0$, (b) $\beta=0.302, \lambda=0.15$ when $\alpha=0.05$, (c) $\beta=0.307, \lambda=0.22$ when $\alpha=0.1,(\mathrm{~d}) \beta=0.3, \lambda=0.17$ when $\alpha=0.2$. The data is the cumulative number of confirmed cases of Wuhan. The values of other parameters are set to: $\gamma_{1}=\frac{1}{21}, \gamma_{2}=\frac{1}{10}$, $\gamma_{3}=\frac{1}{15}, \delta=0.03, l=0.0083$.

reproduction number $R_{0}$ is $3.05 / 3.18 / 3.41 / 3.66$ when $\alpha=0 / 0.05 / 0.1 / 0.2$ by computing the expression $R_{0}=\alpha \beta / \gamma_{1}+(1-\alpha) \beta / \gamma_{2}$. In addition, we find that there is a difference of $A(t)$ in Fig. 2(a),(b),(c) and (d). Next, we will further study the effect of asymptomatic individuals.

We explore how the asymptomatic individuals affect cumulative number $C_{I}(t)$ of individuals in the $I$ state, the number $L(t)$ of individuals in the $L$ state and the number $A(t)$ of individuals in the asymptomatic state in Fig. 3. In Fig. 3(a)(b), we choose the estimated parameter values with $\alpha=0$ and $\alpha=0.2$, respectively. As the individuals in the $A$ state have a longer infection period, $C_{I}(t)$ becomes larger with the increasing of $\alpha$. In addition, with the implementing of control measures and the relatively short incubation period of individuals in the $L$ state, the final numbers of individuals in the $L$ state with different $\alpha$ have basically no difference in Fig. 3(c). However, there is 

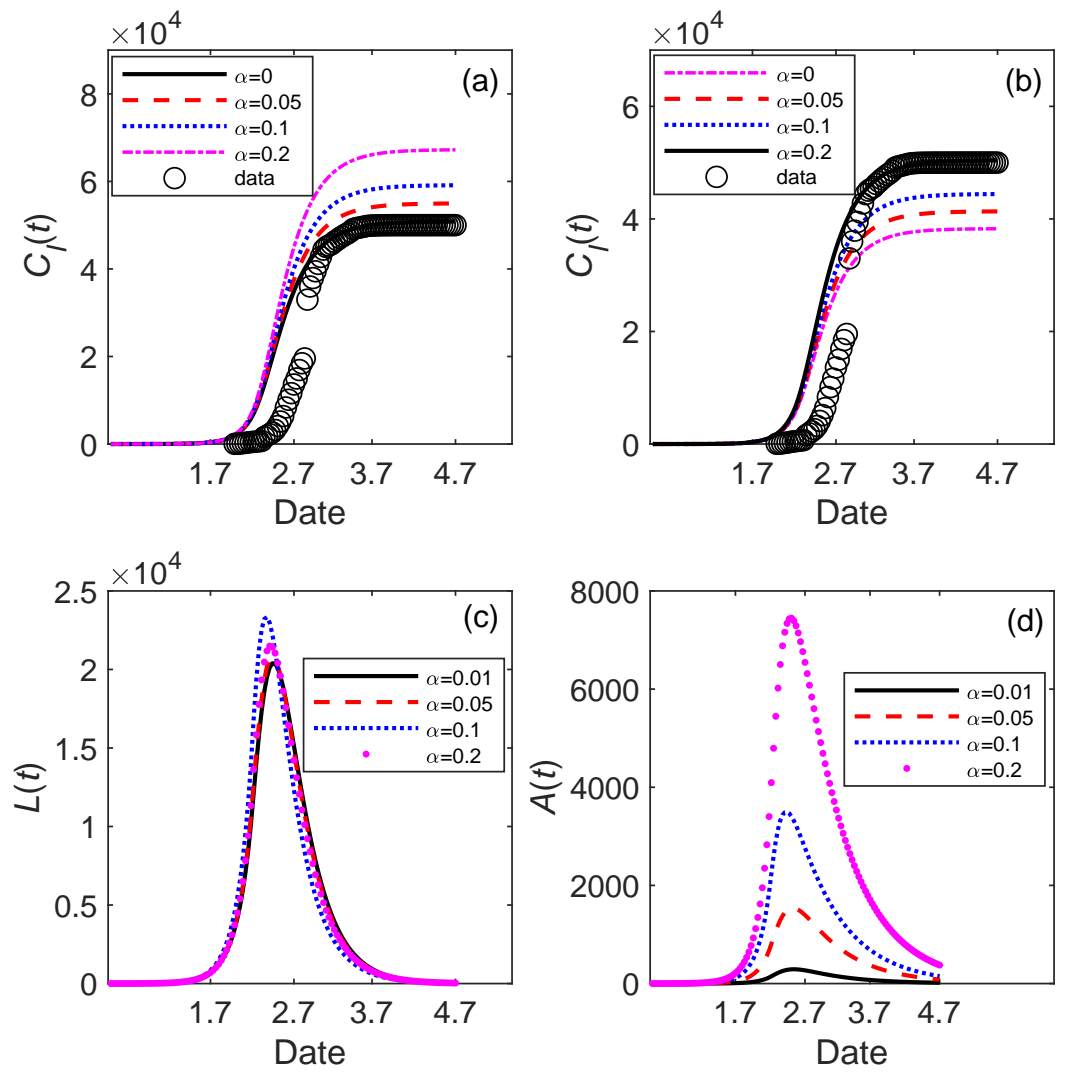

Fig. 3 The change of the number of infected individuals with different $\alpha$. The cumulative number $C_{I}(t)$ of individuals in the $I$ state for different $\alpha$ with the estimated parameters (a) $\beta=0.305, \lambda=0.16$, (b) $\beta=0.3, \lambda=0.17$. The number of individuals in (c) $L$ state and (d) $A$ state with the estimated parameters $\beta=0.302, \lambda=0.14$ when $\alpha=0.01 ; \beta=0.302$, $\lambda=0.15$ when $\alpha=0.05 ; \beta=0.307, \lambda=0.22$ when $\alpha=0.1 ; \beta=0.3, \lambda=0.17$ when $\alpha=0.2$. The data is the cumulative number of confirmed cases of Wuhan. The values of other parameters are set as: $\gamma_{1}=\frac{1}{21}, \gamma_{2}=\frac{1}{10}, \gamma_{3}=\frac{1}{15}, \delta=0.03, l=0.0083$.

still a certain number of asymptomatic individuals in the city when $\alpha$ is larger in Fig. 3(d). Thus, the city still needs to keep a certain strength of control measures after the city was unsealed to avoid the outbreak of COVID-19 again.

After the city was unsealed on April 8, 2020, we will further study the critical strength of control measures to contain the spreading of COVID-19. According to Eq. (3), we can compute $m_{c}$ under different $\alpha$. When $\alpha=0.05(\alpha=$ $0.2)$, the estimated spreading probability is $\beta=0.302(\beta=0.3)$, and thus $m_{c}=0.3139\left(m_{c}=0.2732\right)$. Next, to measure the accuracy of critical strength analysis, we will choose the adjacent values of $m_{c}$ to study the trend of the fitted epidemic curve. When $\alpha=0.01$, we find that the trend of spreading tends to decline when $m_{0}$ is smaller than $m_{c}$ in Fig. $4(\mathrm{a})$, and to expand when 

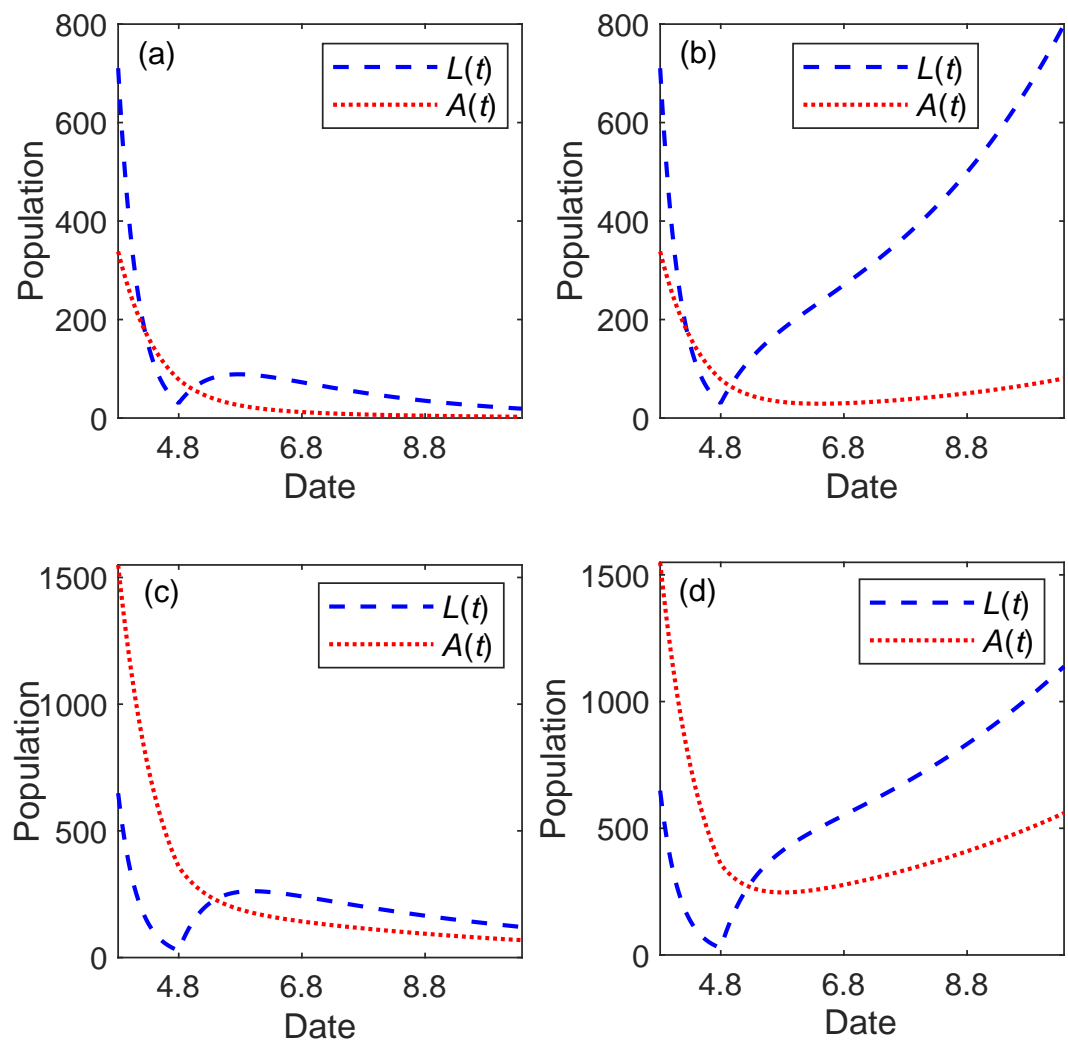

Fig. 4 The change of $A(t)$ and $L(t)$ under different $m_{0}$ after the city was unsealed. $T$ represents the day of April 8, 2020. The temporal trend of the number of individuals in the $A$ and $L$ state when (a) $\alpha=0.05, m_{0}=0.27$, (b) $\alpha=0.05, m_{0}=0.35$, (c) $\alpha=0.2, m_{0}=0.25$, and $(\mathrm{d}) \alpha=0.2, m_{0}=0.3$. The values of other parameters are set to: $\gamma_{1}=\frac{1}{21}, \gamma_{2}=\frac{1}{10}$, $\gamma_{3}=\frac{1}{15}, \delta=0.03, l=0.0083$.

$m_{0}$ is larger than $m_{c}$ in Fig. 4(b). The results are similar when $\alpha=0.2$ in Fig. 4(c)(d).

Besides, we derive the epidemic curve of China except for Hubei province in Fig. 5. The data are the confirmed cases from January 20, 2020, to March 10,2020 . We find that the spreading probability is smaller than that in Wuhan, and is about 0.27 in Fig. $5(\mathrm{a})(\mathrm{c})$. The basic reproduction number $R_{0}$ is $3.03 / 3.31$ when $\alpha=0.1 / 0.2$. With the curve tending to be stable, we compute the critical strength $m_{c}$ of control measures needed when $\alpha=0.1$ and $\alpha=0.2$. The value is 0.33 and 0.30 , respectively. We choose the adjacent values of $m_{c}$ to study the evolution trend of the fitted epidemic curve. When $\alpha=0.1$, we find that the trend of spreading tends to decline when $m_{0}$ is smaller than $m_{c}$, and to expand when $m_{0}$ is larger than $m_{c}$ in Fig. $5(\mathrm{~b})$. The results are similar when $\alpha=0.2$ in Fig. $5(\mathrm{~d})$. 

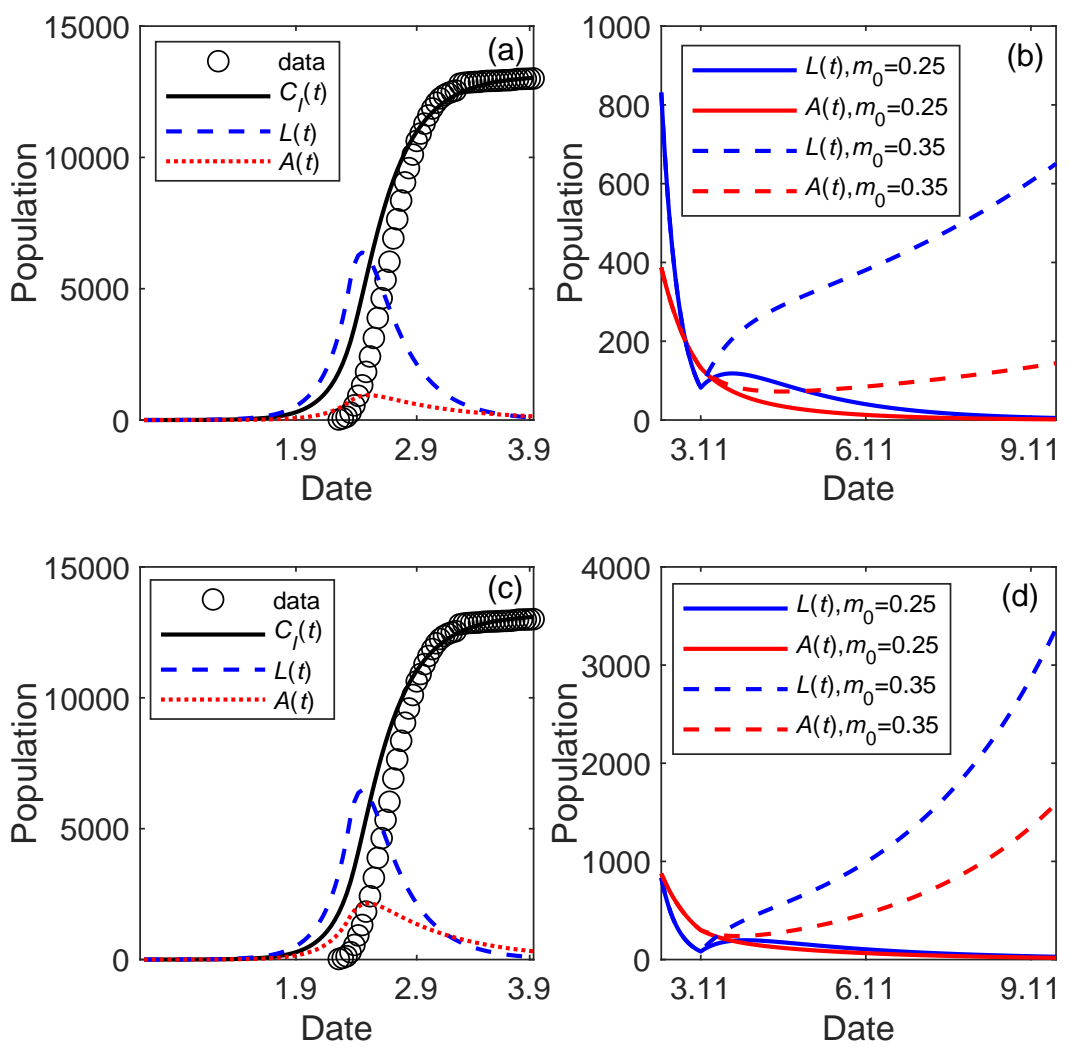

Fig. 5 The fitted epidemic curve with different $\alpha$ and $m_{0}$. The estimated parameters are $\beta=0.273, \lambda=0.29$ when $\alpha=0.1$ in (a)(b), and $\beta=0.271, \lambda=0.3$ when $\alpha=0.2$ in (c)(d). The change of $A(t)$ and $L(t)$ under different $m_{0}$ in (b) and (d), and $T$ represents the day of March 11, 2020. The data is the cumulative number of confirmed cases of China except for Hubei province. The values of other parameters are set as: $\gamma_{1}=\frac{1}{21}, \gamma_{2}=\frac{1}{10}, \gamma_{3}=\frac{1}{15}$, $\delta=0.03, l=0$.

Finally, we explore the relationship between the asymptomatic individuals and critical strength of control measures when there are a few individuals in the $A$ or $L$ state in the early moment of the system in Fig. 6 . In this analysis, we assume that the strength $m(t)$ of the control measures is a fixed value expressed as $m$. When the spreading probability is higher in Fig. 6(b), the critical strength of control measures is smaller than that in Fig. 6(a) which means that the community needs stricter control measures to contain the spreading of the disease. Once the strength of control measures decreases(i.e., $m$ increases) or the probability $\alpha$ for the susceptible individual to be in the $A$ state after infection increases, the total number of infected individuals will increase. Moreover, with $\alpha$ increasing, $m_{c}$ decreases, which signifies that the stricter control measures are needed in the presence of the asymptomatic individuals. 
(a)

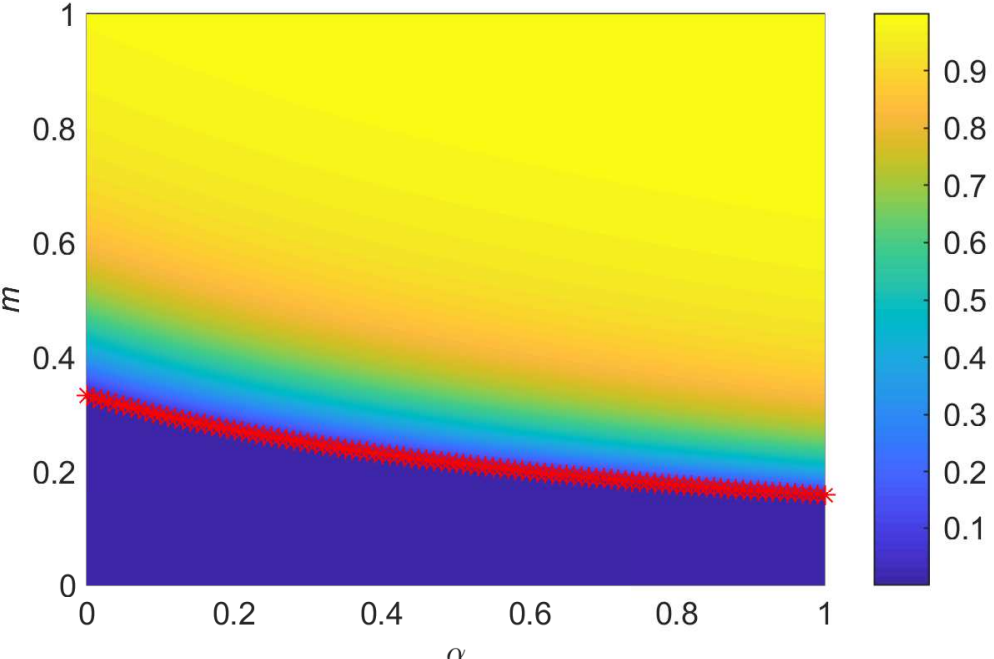

(b)

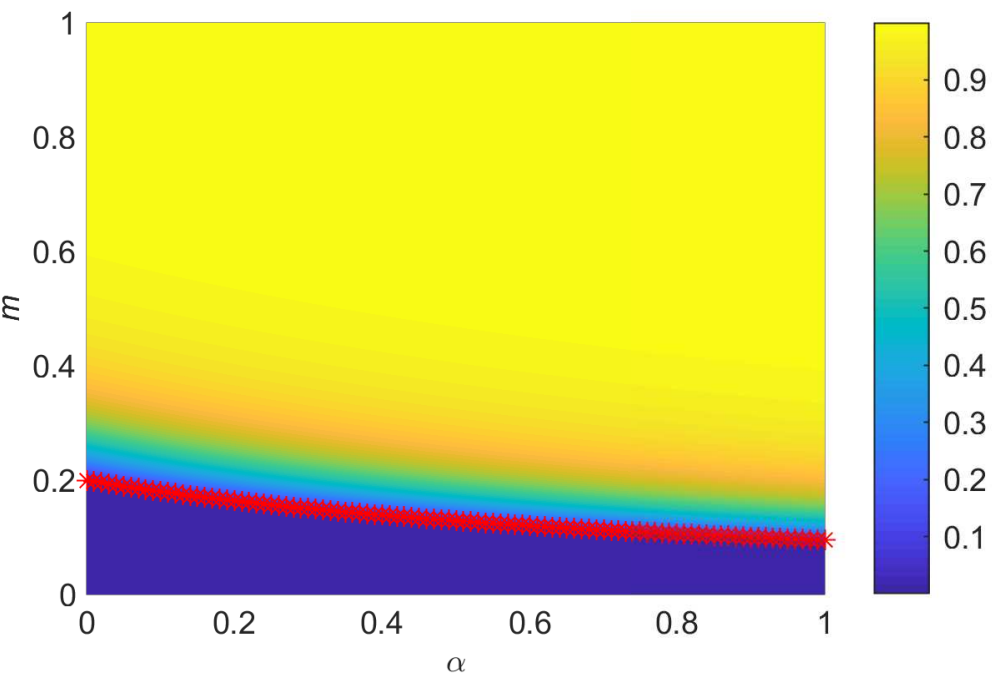

Fig. 6 The total proportion of infected individuals under $(\alpha, m)$. The red asterisks indicate the value of $m_{c}$ under different $\alpha$. The spreading probability is (a) $\beta=0.3$, and (b) $\beta=0.5$. The values of other parameters are set as: $\gamma_{1}=\frac{1}{21}, \gamma_{2}=\frac{1}{10}, \gamma_{3}=\frac{1}{15}, \delta=0.03, l=0$.

\section{Conclusions}

A SALIR model that considers the infection of asymptomatic individuals has been proposed. The critical strength of control measures, which prevents the second outbreak of COVID-19, is analyzed when the spreading of the disease is eased. The result shows that the probability $\alpha$ for a susceptible individual to be asymptomatic after the infection and the duration time $t_{1}$ of infection of asymptomatic individuals all affect the critical strength of control measures. 
The larger the $\alpha$ or $t_{1}$ is, the stricter the adopted control measures should be. Also, based on some clinical characteristics of COVID-19, our model reproduces well the real epidemic curve.

However, there is still a lack of detailed study about the asymptomatic individuals, especially regarding the questions of what the proportion of asymptomatic individuals in population is and how long an asymptomatic individual can carry the virus of COVID-19. Based on these pieces of information, the risk of COVID-19 can be better estimated by using our SALIR model.

In summary, our SALIR model analyzes the risk of the asymptomatic population for the second outbreak of COVID-19. As the suggestion of our model, the authorities should pay close attention to the presence of asymptomatic individuals, including the formulation of public health intervention policy and virus detection of suspected individuals.

\section{Acknowledgments}

This work was supported by the National Natural Science Foundation of China (Grant No. 61932005) and the Fundamental Research Funds for the Central Universities with contract number 2019XD-A10.

\section{Interests Conflict}

The authors declare that they have no conflict of interests.

\section{References}

1. Coronavirus disease 2019 (COVID-19) Situation Report 78 (World Health Organization). URL https://www.who.int/docs/default-source/coronaviruse/ situation-reports/20200407-sitrep-78-covid-19.pdf?sf vrsn=bc43e1b_2

2. W.J. Guan, Z.Y. Ni, Y. Hu, W.H. Liang, C.Q. Ou, J.X. He, L. Liu, H. Shan, C.L. Lei, D.S. Hui, et al., New England Journal of Medicine (2020). DOI 10.1056/ NEJMoa2002032

3. R.M. Anderson, B. Anderson, R.M. May, Infectious diseases of humans: dynamics and control (Oxford university press, 1992)

4. N. Shao, J. Cheng, W. Chen, MedRxiv (2020). DOI 10.1101/2020.02.17.20023747

5. J. Wallinga, M. Lipsitch, Proceedings of the Royal Society B: Biological Sciences 274(1609), 599 (2007)

6. P.P. Shi, S.L. Cao, P.H. Feng, MedRxiv (2020). DOI 10.1101/2020.02.16.20023655

7. Z.F. Yang, Z.Q. Zeng, K. Wang, S.S. Wong, W.H. Liang, M. Zanin, P. Liu, X.D. Cao, Z.Q. Gao, Z.T. Mai, et al., Journal of Thoracic Disease 12(3), 165 (2020)

8. Coronavirus disease 2019 (COVID-19) Situation Report - 73 (World Health Organization). URL https://www.who.int/docs/default-source/coronaviruse/ situation-reports/20200402-sitrep-73-covid-19.pdf?sf vrsn=5ae25bc7_6

9. Y. Bai, L. Yao, T. Wei, F. Tian, D.Y. Jin, L. Chen, M. Wang, JAMA 323(14), 1406 (2020). DOI 10.1001/jama.2020.2565

10. W. Wang, J. Tang, F. Wei, Journal of Medical Virology 92(4), 441 (2020). DOI 10.1002/jmv.25689

11. W.J. Guan, Z.Y. Ni, Y. Hu, W.H. Liang, C.Q. Ou, J.X. He, L. Liu, H. Shan, C.L. Lei, D.S. Hui, et al., Science (2020). DOI 10.1126/science.aba9757 
12. Situation Reports from National Health Commission of the People's Republic of China(in Chinese) (National Health Commission of the People's Republic of China). URL https://www.nhc.gov.cn/xcs/yqtb/list_gzbd.shtml

13. Q. Li, X. Guan, P. Wu, X. Wang, L. Zhou, Y. Tong, R. Ren, K.S. Leung, E.H. Lau, J.Y. Wong, et al., New England Journal of Medicine 382(13), 1199 (2020). DOI 10.1056/NEJMoa2001316

14. Baidu Qianxi(in Chinese) 2020. URL https://qianxi.baidu.com/

15. M. Chinazzi, J.T. Davis, C. Gioannini, M. Litvinova, A. Pastore y Piontti, L. Rossi, X. Xiong, M.E. Halloran, I. Longini, A. Vespignani, Laboratory for the Modeling of Biological Socio-technical Systems (2020). URL https://www.mobs-lab.org/uploads/ 6/7/8/7/6787877/wuhan_novel_coronavirus__6_.pdf

16. N. Imai, I. Dorigatti, A. Cori, S. Riley, N. Ferguson, (2020). DOI 10.25561/77149 
Figures

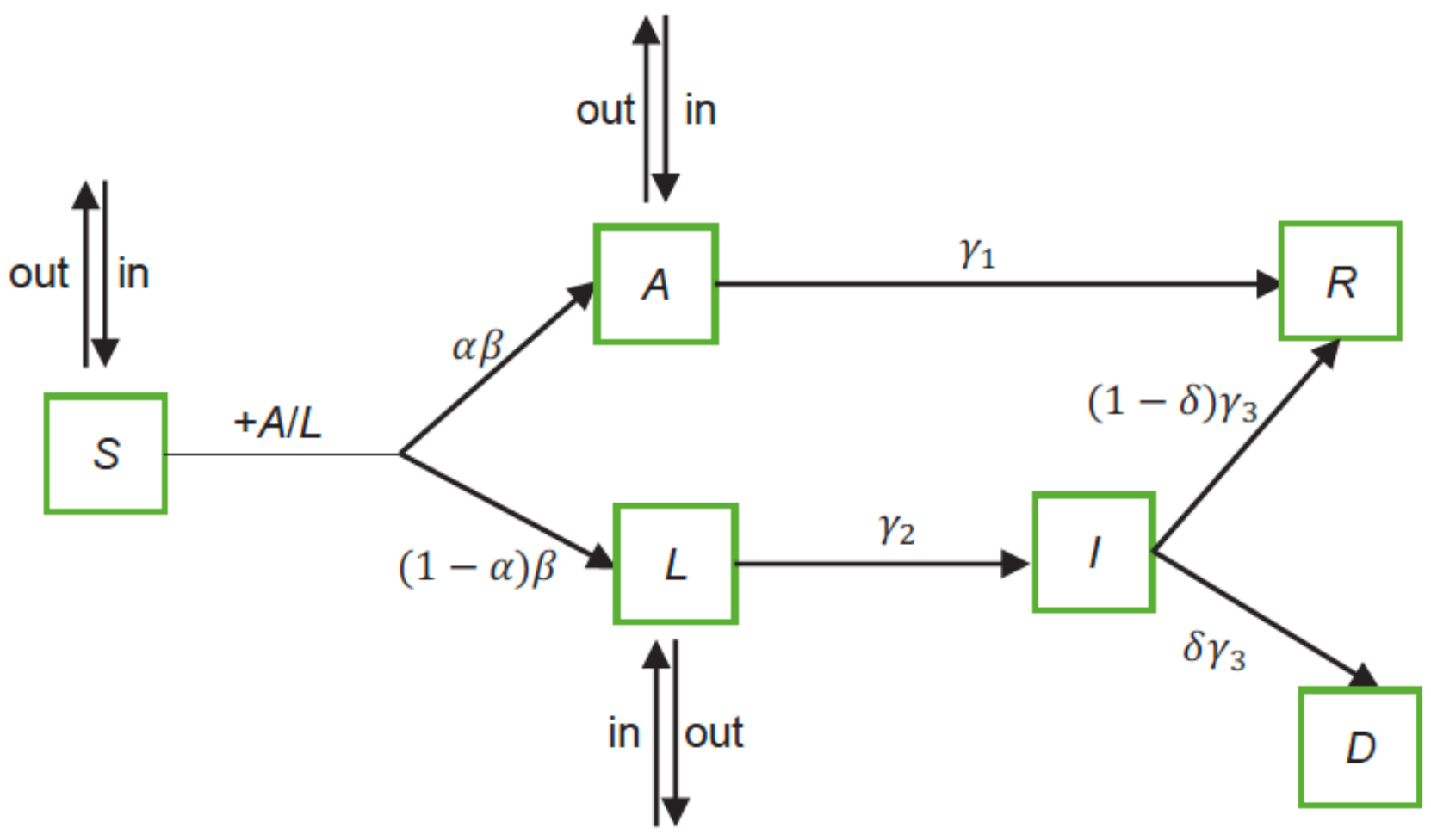

Figure 1

The sketch map of the SALIR model. 

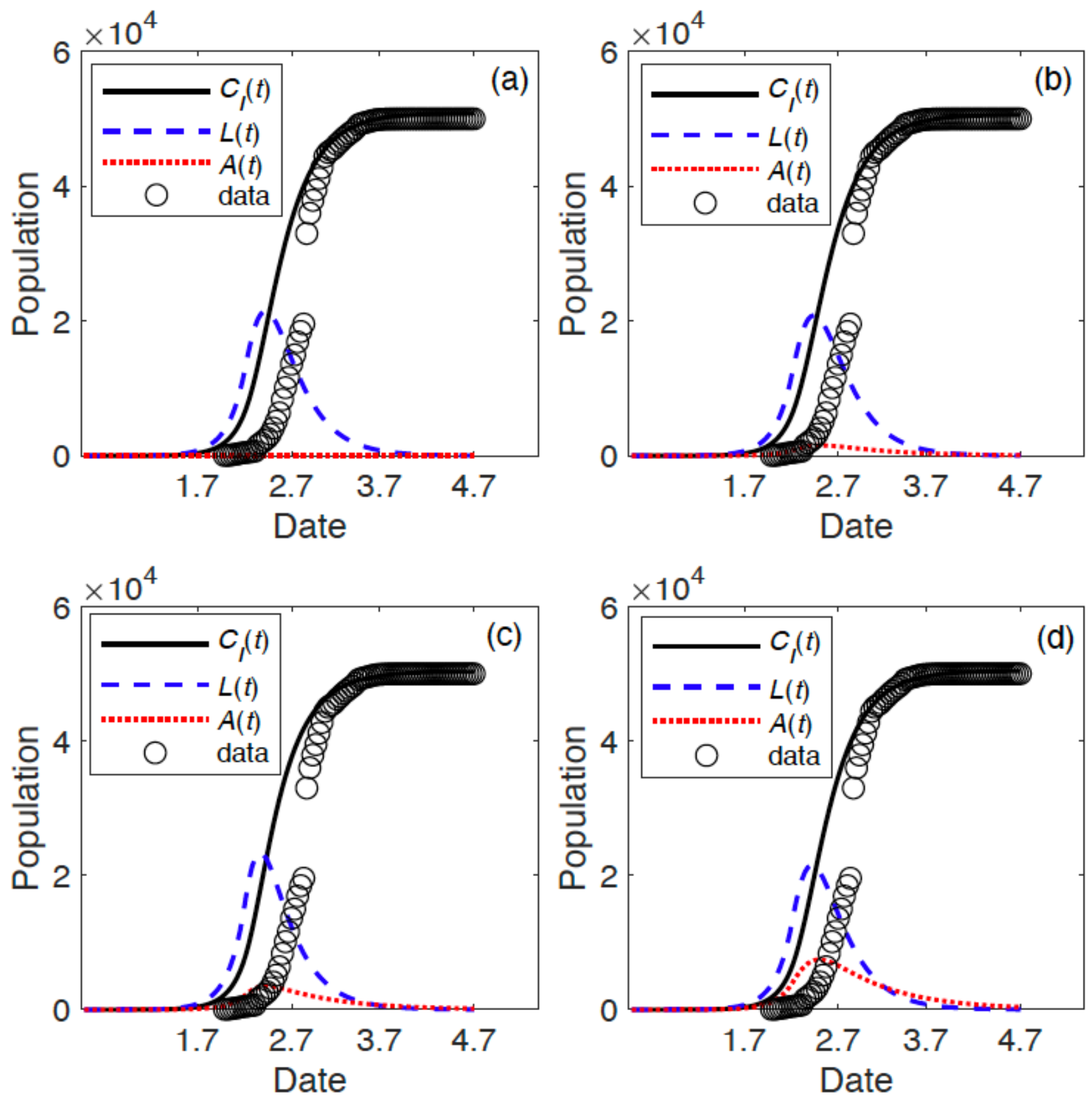

Figure 2

The fitted epidemic curve with different $\$ \backslash$ alpha's $\$$. The estimated parameters are $(a) \$ \backslash$ beta $=0.305 \$$, $\$ \backslash$ lambda $=0.16 \$$ when $\$ \backslash$ alpha $=0 \$,(b) \$ \backslash b e t a=0.302 \$$, $\$ \backslash$ lambda $=0.15 \$$ when $\$ \backslash$ alpha $=0.05 \$$, (c) $\$ \backslash$ beta $=0.307 \$$, $\$ \backslash$ lambda $=0.22 \$$ when $\$ \backslash a \mid p h a=0.1 \$$, (d) $\$ \backslash$ beta $=0.3 \$$, $\$ \backslash$ lambda $=0.17 \$$ when $\$ \backslash$ alpha $=0.2 \$$. The data is the cumulative number of confirmed cases of Wuhan. The values of other parameters are set to: $\$ \backslash$ gamma_ $\{1\}=\backslash$ frac $\{1\}\{21\} \$$, $\$$ gamma_ $\{2\}=\backslash$ frac $\{1\}\{10\} \$$, $\backslash \backslash$ gamma_ $\{3\}=\backslash$ frac $\{1\}$ $\{15\}$, $\$ \backslash$ delta=0.03\$, $\$ 1=0.0083 \$$. 

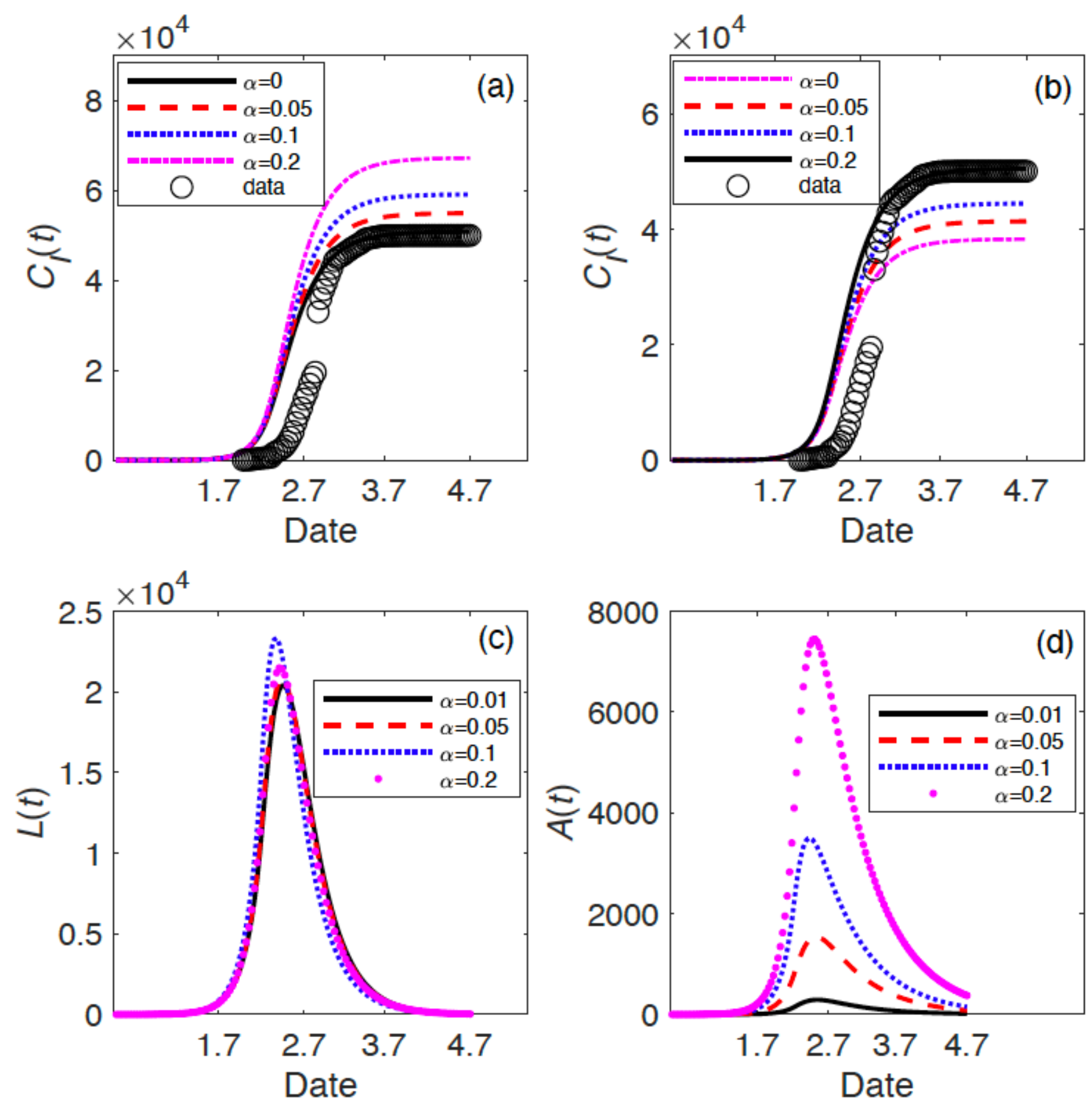

Figure 3

The change of the number of infected individuals with different $\$ \backslash$ alpha\$. The cumulative number $\$ C_{-}\{1\}$ $(\mathrm{t}) \$$ of individuals in the $\$ \mathbf{S}$ state for different $\$ \backslash$ alpha $\$$ with the estimated parameters (a) $\$$ beta $=0.305 \$$, $\$ \backslash$ lambda=0.16\$, (b) $\$$ beta $=0.3 \$$, $\$ \backslash$ lambda $=0.17 \$$. The number of individuals in (c) $\$ L \$$ state and (d) $\$ A \$$ state with the estimated parameters $\$ \backslash$ beta $=0.302 \$$, $\$ \backslash$ lambda $=0.14 \$$ when $\$ \backslash$ alpha $=0.01 \$ ;$ $\$ \backslash$ beta $=0.302 \$$, $\$ \backslash$ lambda $=0.15 \$$ when $\$ \backslash a|p h a=0.05 \$ ;|$ beta $=0.307 \$$, $\$ \backslash$ lambda $=0.22 \$$ when $\$ \backslash$ alpha $=0.1 \$ ;\rangle$ beta $=0.3 \$$, $\$ \backslash$ lambda $=0.17 \$$ when $\$ \backslash$ alpha $=0.2 \$$. The data is the cumulative number of confirmed cases of Wuhan. The values of other parameters are set as: $\$$ Igamma_ $\{1\}=\backslash$ frac $\{1\}\{21\} \$$,

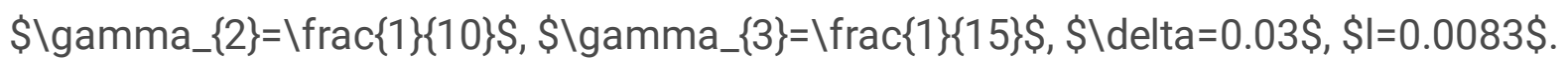



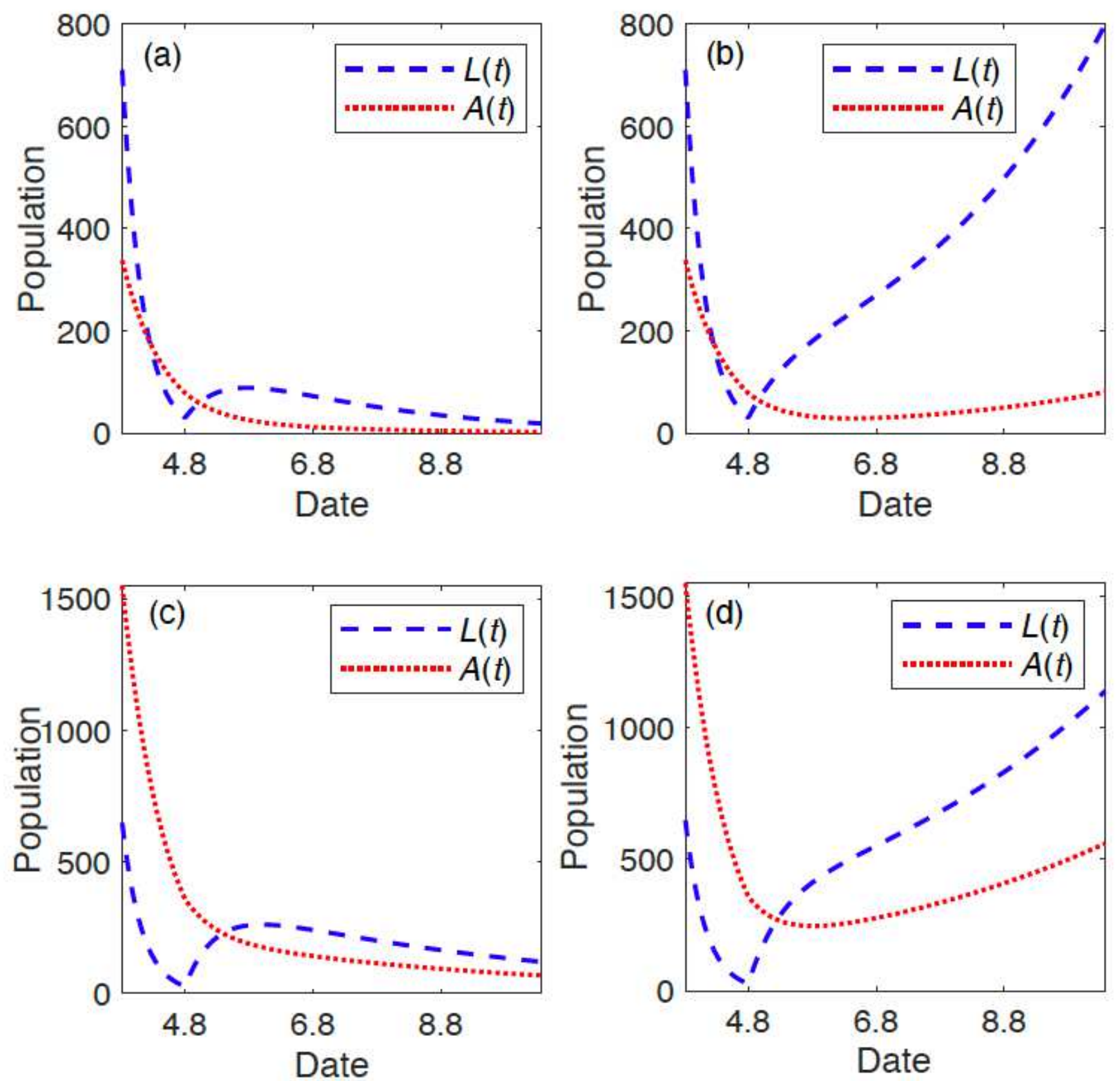

Figure 4

The change of $\$ A(t) \$$ and $\$ L(t) \$$ under different $\$ m_{-}\{0\} \$$ after the city was unsealed. $\$ T \$$ represents the day of April 8, 2020. The temporal trend of the number of individuals in the $\$ A \$$ and $\$ L \$$ state when (a) $\$ \backslash$ alpha $=0.05 \$, \$ m_{-}\{0\}=0.27 \$$, (b) $\backslash \backslash$ alpha $=0.05 \$, \$ m_{-}\{0\}=0.35 \$$, (c) $\backslash \backslash$ alpha $=0.2 \$, \$ m_{-}\{0\}=0.25 \$$, and (d) $\$ \backslash$ alpha $=0.2 \$, \$ m_{-}\{0\}=0.3 \$$. The values of other parameters are set to: $\$ \backslash$ gamma_ $\{1\}=\backslash$ frac $\{1\}\{21\} \$$,

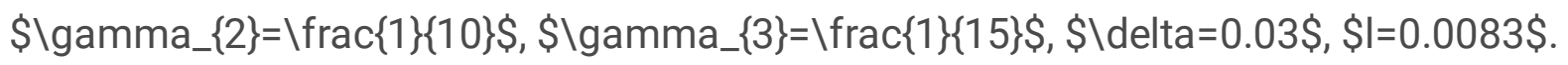



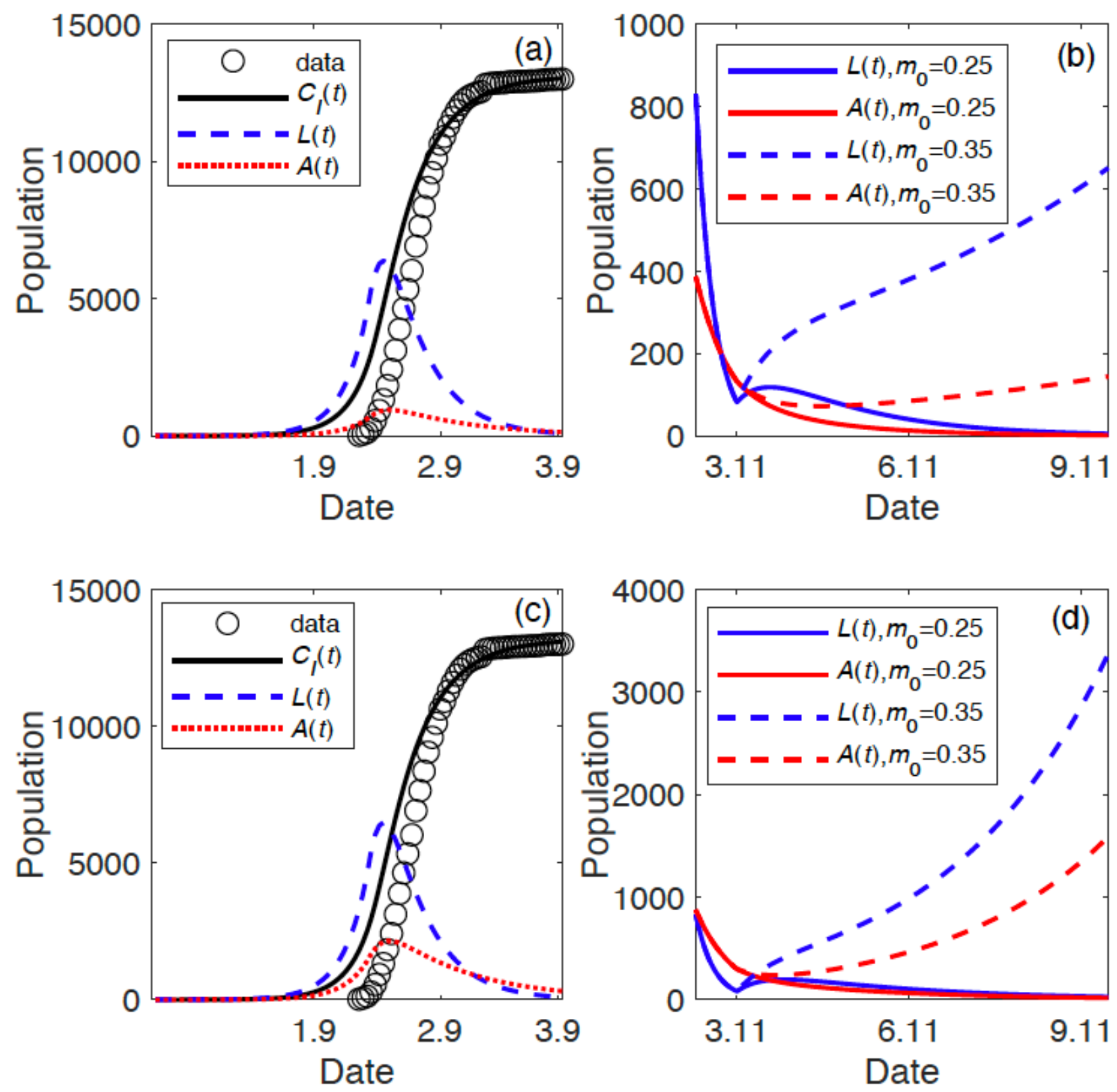

Figure 5

The fitted epidemic curve with different $\$ \backslash$ alpha $\$$ and $\$ m_{-}\{0\} \$$. The estimated parameters are $\$ \backslash$ beta $=0.273 \$$, $\$ \backslash$ lambda $=0.29 \$$ when $\$ \backslash$ alpha $=0.1 \$$ in $(a)(b)$, and $\$ \backslash$ beta $=0.271 \$$, $\$ \backslash$ lambda $=0.3 \$$ when

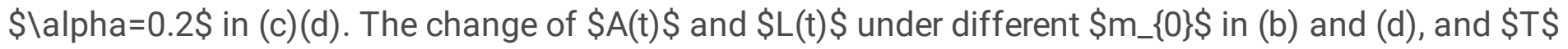
represents the day of March 11, 2020. The data is the cumulative number of confirmed cases of China except for Hubei province. The values of other parameters are set as: $\$ \backslash$ gamma_ $\{1\}=\backslash$ frac $\{1\}\{21\} \$$,

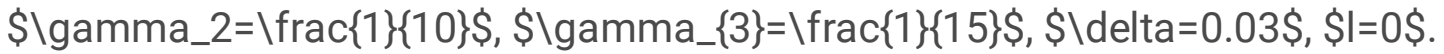


(a)

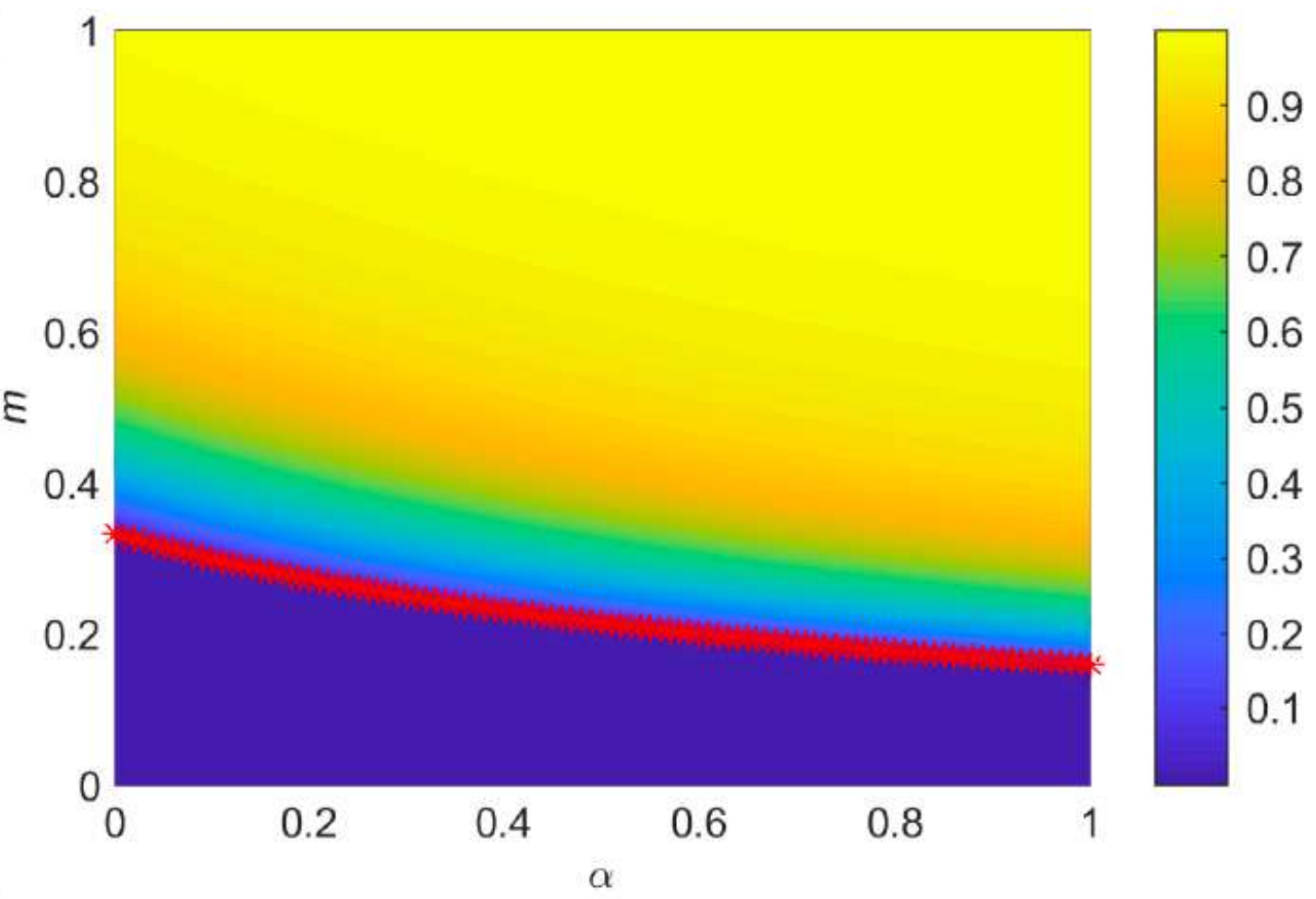

(b)

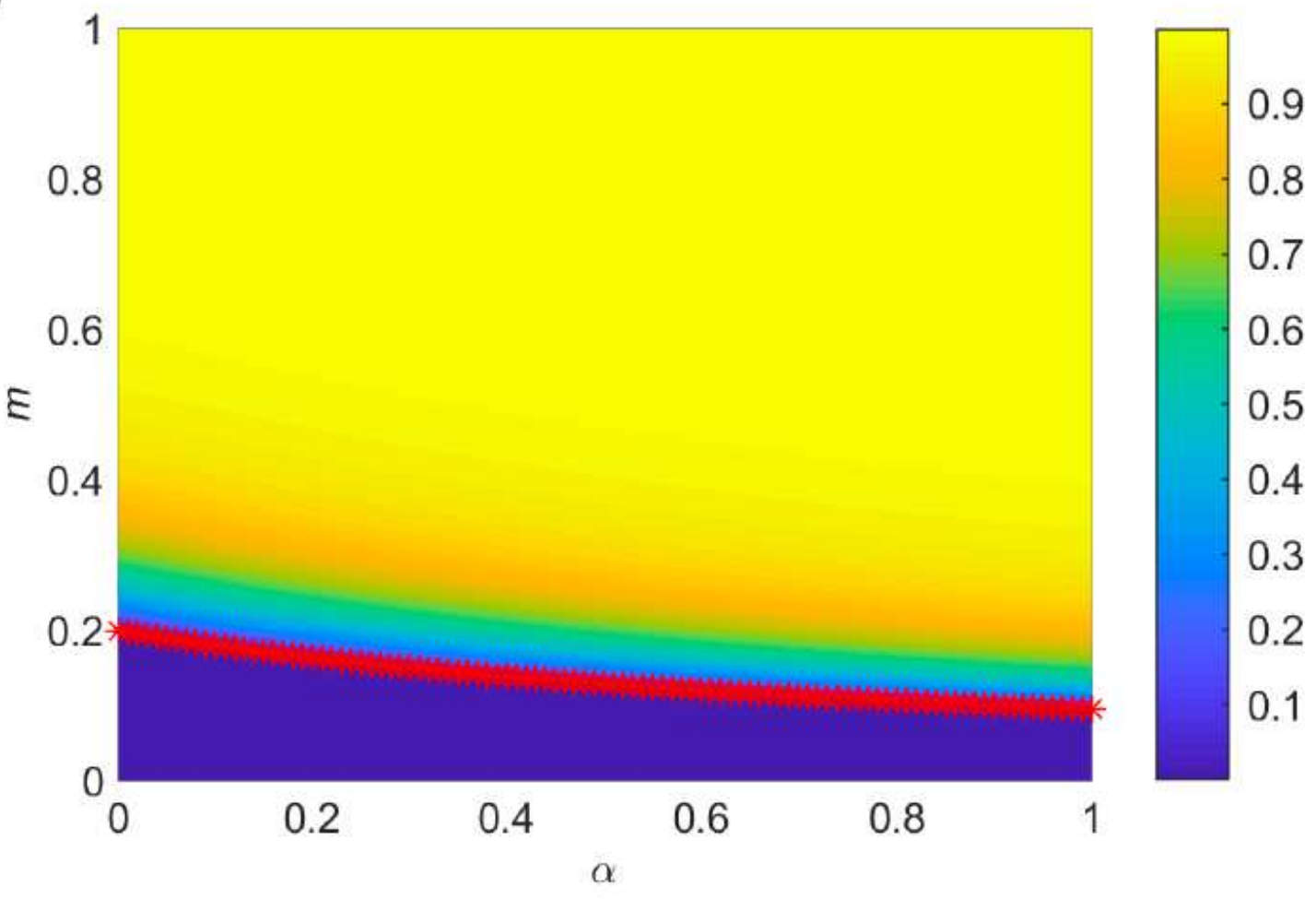

\section{Figure 6}

The total proportion of infected individuals under $\$(|a| p h a, m) \$$. The red asterisks indicate the value of $\$ m_{-}\{c\} \$$ under different $\$ \backslash$ alpha $\$$. The spreading probability is (a) $\$ \backslash$ beta $=0.3 \$$, and (b) $\$ \backslash b e t a=0.5 \$$. The values of other parameters are set as: $\$ \backslash$ gamma_ $\{1\}=\backslash$ frac $\{1\}\{21\} \$$, $\$$ Igamma_ $\{2\}=\backslash f r a c\{1\}\{10\} \$$, $\$$ Igamma_ $\{3\}=\backslash$ frac $\{1\}\{15\} \$$, $\$ \backslash$ delta $=0.03 \$, \$=0 \$$. 


\section{Supplementary Files}

This is a list of supplementary files associated with this preprint. Click to download.

- data.xls

- SALIR.m 